\title{
Evaluation of Neurological Disorder Using Computed Tomography and Magnetic Resonance Imaging
}

\author{
Saeed M. Bafaraj \\ Department of Diagnostic Radiology, Faculty of Applied Medical Sciences, King Abdulaziz University, Jeddah, KSA \\ Email: smbafaraj@kau.edu.sa
}

How to cite this paper: Bafaraj, S.M. (2021) Evaluation of Neurological Disorder Using Computed Tomography and Magnetic Resonance Imaging Journal of Biosciences and Medicines, 9, 42-51.

https://doi.org/10.4236/jbm.2021.92005

Received: January 5, 2021

Accepted: February 20, 2021

Published: February 23, 2021

Copyright (๑) 2021 by author(s) and Scientific Research Publishing Inc. This work is licensed under the Creative Commons Attribution International License (CC BY 4.0).

http://creativecommons.org/licenses/by/4.0/

\section{(c) (i) Open Access}

\begin{abstract}
Background: The integration of different imaging technologies such as computed tomography (CT) and magnetic resonance imaging (MRI) assists clinicians in diagnosing several neurological diseases at an initial stage, thereby inhibiting their development into serious medical conditions. With respect to the severity of neurological diseases and the increasing popularity of imaging tools, the current study has evaluated the findings of CT and MRI in examining neurological disorders. Methods. A retrospective study was conducted at the faculty of applied medical sciences, King Abdul-Aziz University from September 2017 to 2018 including details of 43 patients with complaints of potential neurological disturbances such as headache, oligodendroglioma, CNS infiltration, hypoxia, weakness, epilepsy and multiple sclerosis. The history of patients including their age, gender, reason for examination and findings of CT and MRI was evaluated in the study. Results. The results showed that patients in the age group of 21 to 40 years were more prone to developing neurological disorders. The major reasons for neurological examination in individuals were found to be headaches $(n=7)$, multiple sclerosis $(n=5)$ and epilepsy $(n=3)$. The evaluation of patients' records revealed that only 4 patients underwent CT scans; however, all of them went through the MRI. Statistically significant results were obtained for the patients scanned and examined through MRI rather than CT. Conclusions. The results revealed MRI as more successful in diagnosing different neurological conditions in comparison with CT scan. The study further suggests that MRI can be used as an integral diagnostic approach for neurological understanding of major diseases of the central nervous system.
\end{abstract}

\section{Keywords}

Neurological Disorder, Computed Tomography, Magnetic Resonance 


\section{Introduction}

The accurate and precise diagnosis of a neurological disease ensures the provision of adequate and timely neurological care and treatment. However, the intricate association of neurological disturbances with the complex nervous system is the most challenging factor in the diagnosis, management and treatment of these diseases. In this context, development of modern imaging technology to diagnose neurological diseases has contributed a lot to the provision of optimal neurologic care [1]. Disorders of the nervous system are associated with structural, biochemical and electrical abnormalities in nerves, spinal cord and the brain that are manifested in the form of a range of symptoms such as headache/migraine, dizziness and vertigo. According to Siuly and Zhang (2016), the nervous system is vulnerable to 600 diseases approximately including Alzheimer's disease, epilepsy, stroke, migraine, Parkinson's disease, multiple sclerosis, brain tumors and brain trauma [2].

The use of radiological imaging in medical care dates back to 1895 when X-rays were discovered by the Nobel Laureate Wilhelm Conrad Rontgen [3]. Since then, the conventional radiology $(\mathrm{Rx})$ has been upgraded with the development of various other body imaging techniques to diagnose complex neurological disorders. For instance, the advent of diagnostic imaging tools such as computed tomography (CT), magnetic resonance imaging (MRI) and nuclear medicine, has contributed significantly to the diagnoses of neurological diseases as these tools provide three-dimensional anatomy of the human body. Moreover, these techniques can help identify various malignancies, which, in turn, assist in determining the required course of action and the right therapeutic approach.

MRI is considered one of the best imaging techniques for the optimal management of secondary headaches [4]. The discrimination between primary and secondary types of headaches becomes difficult and it has been a diagnostic dilemma for healthcare professionals. Primary headaches have no underlying organic disease as its root cause; however, a neurological disorder is the root cause of secondary headaches. These organic diseases may range from extracranial benign conditions to life-threatening intracranial pathological conditions [5]. A previous study evaluated the clinical overt incidence like stroke, myocardial infarction and death during follow-up [6]. These results were useful in assessing the growth of disease, knowledge of the disease and understanding the complex disease development processes. Previous studies conducted by Jindal et al. (2016) and Gao and Jiang (2013) reported both CT and MRI to be effective in diagnosing major neurological disturbances [7] [8].

MRI findings are used to assess cerebrovascular impairment as well as the possible cause of a neurological disorder [9]. An understanding of the multifaceted neurobiological changes causing neurological disorders is likely to expand on account of current advancements in MRI and other imaging modalities. Moreover, a deep understanding of these techniques may provide further insights for developing new neuroimaging tools [5]. CT scan and MRI are two tra- 
ditional imaging techniques used for identification of neurological disorders. However, MRI is a more effective technique in comparison with CT scan, given its advantages of better integration of soft tissues, strengthened contrast enhancement, decreased bone artifacts, partial volume effects and direct multi-planar imaging scan [10]. It has been shown that MRI is useful in detecting acute onset of the worst headache triggering immediate referral for an intracranial aneurysm [11]. On account of its efficiency and practicality, CT scan plays an important role in the diagnosis of strokes.

The neurological and physical examination provides detailed clinical history that assists in making a decision about choosing MRI or CT scan to find the underlying brain pathology. MRI or CT scan helps in allaying the anxiety of patients and their family members despite the absence of Clinical Warning Criterion (CWC). In case of children and pregnant and child-bearing females, the issue of appropriate radiation dose required for neuroimaging comes to the fore. Therefore, in many cases, there is a need to balance the use of CT scan and radiation dose. There are limited studies that have evaluated the findings of CT scan and MRI in patients with neurological complaint mainly headache. Therefore, the current study aims to evaluate the findings of CT and MRI in examining neurological disorders in patients with respect to the severity of the neurological diseases and the increasing popularity of the imaging tools.

\section{Materials and Methods}

\subsection{Study Design}

A retrospective observational study was conducted for patients who had undergone CT scan or MRI to identify possible neurological disorders in 43 selected patients who complain of headache, bracket age of 1 to 80 years. The study was conducted in the time period of 10 months from September 2017 to June 2018. Ethical approval was acquired from the Faculty of Applied Medical Sciences, King Abdulaziz University after providing a detailed description of the study before conducting it.

\subsection{Inclusion and Exclusion Criteria}

The inclusion criteria for this study were patients with main complaint of headache (otherwise healthy individual), with complete CT or MRI study reports with good quality images. However, patients with history of neurosurgery or acute head injury were excluded. Moreover, the patients were eligible if they were reported with either one of the neurological disorders, and were examined via either MRI or CT. Patients were excluded on the basis of their age alone. Likewise, the patients were assessed by an experienced stroke neurologist at examination.

\subsection{Data Collection}

The medical records of patients were studied to highlight the occurrence of po- 
tential neurological disturbances such as headache, oligodendroglioma, CNS infiltration, hypoxia, weakness, epilepsy and multiple sclerosis. The detailed history of patients pertaining to age, gender and reason for examination was also collected. Finally, the findings of CT scan and MRI were evaluated.

\subsection{Data Analysis}

The data collected were analyzed by using Microsoft Excel software. Descriptive statistics were used to present the study results.

\section{Results}

The demographic analysis of the participating patients showed that the number of male and female patients was almost similar (21 males and 22 females) in the study. The age group in which the episodes of neurological disorders were found to be frequent was 21 to 40 years $(n=15)$. Major reasons for examination of individuals were found to be: headaches $(n=8)$, multiple sclerosis $(n=5)$, and epilepsy $(\mathrm{n}=3)($ Table 1$)$.

The evaluation of patient's records revealed that only a few patients $(n=4)$ underwent CT scans; whereas, MRI scan was performed in all patients (Table 2). Table 3 shows the findings of CT scans and MRI, which shows that MRI was

Table 1. Demographic profile of respondents.

\begin{tabular}{|c|c|c|}
\hline Measure & Items & Frequency \\
\hline \multirow{2}{*}{ Gender } & Male & 21 \\
\hline & Female & 22 \\
\hline \multirow{5}{*}{ Age } & $<1$ year & 1 \\
\hline & $1-20$ years & 10 \\
\hline & 21 - 40 years & 15 \\
\hline & $41-60$ years & 14 \\
\hline & $61-80$ & 3 \\
\hline \multirow{12}{*}{ Reason of examination } & Bilateral optic neuritis and anterior uveitis & 1 \\
\hline & Brainstem lesions & 1 \\
\hline & CA right breast & 1 \\
\hline & Chordoma & 1 \\
\hline & CNS lymphoma & 1 \\
\hline & Diabetes mellitus & 1 \\
\hline & Epilepsy & 3 \\
\hline & Global developmental delay & 1 \\
\hline & Headache & 8 \\
\hline & Hemorrhagic leukemic CNS infiltration & 1 \\
\hline & Hydrocephalus & 1 \\
\hline & Hypoxia & 1 \\
\hline
\end{tabular}




\section{Continued}

$\begin{array}{cl}\text { Intraventricular tumor } & 1 \\ \text { Kidney evaluation } & 1 \\ \text { Left MTS } & 1 \\ \text { Meningoencephalitis } & 1 \\ \text { Multiple sclerosis } & 5 \\ \text { Multiple brain meningioma post resection } & 1 \\ \text { Oligodendroglioma } & 1 \\ \text { Paracellar mass } & 1 \\ \text { Weakness } & 1 \\ \text { Tuberous sclerosis } & 1 \\ \text { Surgical resection } & 1 \\ \text { Vasculitis } & 2 \\ \text { Post posterior fossa tumor resection } \\ \text { Residual and the size of the non-operated tumor } \\ \text { Post meningioma resection and post } \\ \text { evacuation of hematoma } \\ \text { RT upper limb weakness }\end{array}$

Table 2. Performance of CT and MRI.

\begin{tabular}{ccc}
\hline & Variable & Frequency \\
\hline \multirow{2}{*}{ CT Scan } & Yes & 4 \\
& No & 39 \\
& No scanning & 0 \\
\hline \multirow{2}{*}{ MRI } & Yes & 43 \\
& No & 0 \\
& No scanning & 0 \\
\hline
\end{tabular}

Table 3. Findings of CT scan and MRI.

\begin{tabular}{llcc}
\hline \multicolumn{1}{c}{ Variable } & Frequency & p-value \\
\hline \multirow{2}{*}{ CT findings neuro-navigation } & 1 & 0.31 \\
& Features of Dandy-Walker malformation & 1 & \\
& No acute brain insult & 4 & \\
& Left parietal sub-acute infarction & 1 & \\
& Signs of interval worsening with development of & 1 & \\
& acute hydrocephalus & 1 & 0.00 \\
& Interval development of irregular enhancing lesion & 1 & \\
& Agenesis of the corpus callosum & 1 & \\
BRI Findings & & \\
& Bilateral subcortical, deep white matter, and & 1 & \\
\hline
\end{tabular}




\section{Continued}

\begin{tabular}{lc} 
Global brain incomplete maturation & 1 \\
$\begin{array}{l}\text { Third ventricular colloid cyst with no significant } \\
\text { hydrocephalus }\end{array}$ & 1 \\
$\begin{array}{l}\text { Interval development of right mesiotemporal } \\
\text { enhancing focus }\end{array}$ & 1 \\
Interval reduction in size and enhancement of the & \\
previously seen multiple cortical based low T2 & 2 \\
signal intensity lesions & \\
Ischemic disease & 2 \\
Clival chordoma & 1 \\
$\begin{array}{l}\text { No enhancing cerebral or cerebellar masses } \\
\text { suggestive of metastasis }\end{array}$ & 1 \\
$\begin{array}{l}\text { No evidence of acute infarction, intra or extra-axial } \\
\text { hemorrhage }\end{array}$ & 1 \\
Normal MR features of the brain & 2 \\
$\begin{array}{l}\text { Posterior fossa cystic structure likely representing } \\
\text { of Dandy-Walker malformation }\end{array}$ & 1 \\
Large heterogeneously enhancing right parafalcine \\
mass likely representing tumor recurrence \\
$\begin{array}{l}\text { Relative decrease size with associated relative } \\
\text { increased signal intensity of left hippocampus } \\
\text { Right frontal lobe meningioma resection }\end{array}$ & 1 \\
Lissencephaly-pachygyria spectrum & 2 \\
Unremarkable MR features of the brain & \\
\hline
\end{tabular}

more successful in diagnosing major and most of the neurological issues in patients; on the other hand, CT scan was able to diagnose successfully only a few neurological disturbances. MRI findings majorly revealed unremarkable MR features of the brain $(n=13)$, followed by interval reduction in size and enhancement of the previously seen multiple cortical based low T2 signal intensity lesions $(\mathrm{n}=2)$, Ischemic disease $(\mathrm{n}=2)$, normal MR features of the brain $(\mathrm{n}=$ $2)$, right frontal lobe meningioma resection $(\mathrm{n}=2)$, and Lissencephaly-pachygyria spectrum $(n=2)$. The overall findings of the MRI scan were significant based on the achieved p-value (0.00) whereas CT scan showed no significance based on the achieved p-value (0.31).

\section{Discussion}

The current study has assessed the detection of a number of neurological disturbances in patients with a wide range of symptoms by using CT and MRI as the imaging techniques. The study findings showed that MRI contributes significantly to the diagnosis of neurological disturbances in comparison with CT. A similar study was conducted by Clayton (2016), who used MRI to assess neurological disorders in patients and showed that men and women suffering from migraine 
demonstrate differences in brain structure and connectivity [12]. Another study conducted by Liu et al. (2011) proved MRI to be very effective in detecting chronic migraine and neurological dysfunction in affected individuals [13]. Clinicians are likely to face constant confrontations on the issue of importance of confirming the presence of neurological disorders; however, it is not possible to justify the presence of severe diseases without any effective and accepted clinical evidence.

The current study reported detection of multiple sclerosis or epilepsy in the recruited participants with the help of CT scan and MRI. Such findings have also been reported by Medvid et al. (2015) who suggested that MRI provided better results during examination of neurological diseases in contrast to other evaluation techniques such as CT scan [14]. Headache has also been reported as one of the major symptoms of neurological disorders in the current study. These findings are consistent with those obtained from the study conducted by Holle and Obermann (2013) who diagnosed headache-related disorders using neuroimaging technology [15]. Another study conducted by Nagahama et al. (2017) reported remarkable results obtained from the use of MRI during their assessment of patients suffering from Rasmussen syndrome [16]. Degnan and Levy (2011) also showed MRI as a better neuroimaging tool [17]. Similarly, the current study has also found that MRI detected one sided lateralization in majority of the patients which is considered as another advantage of MRI over CT neuroimaging.

A previous study conducted by Aygun and Bildik (2003) investigated the incidence of intracranial pathology using CT to determine the importance of clinical warning criteria for the prediction of a possible lesion [18]. The results revealed that CT scans revealed possible reasons that might cause headaches. There was an increase in the rate of detection of positive findings in patients who met the clinical warning criteria [18]. A recent study conducted by Rai et al. (2016) has evaluated the findings of CT and MRI in patients with complaints of headache [5]. The results showed that these techniques were effective in diagnosing pathology of brain parenchyma, sinusitis, chronic suppurative otitis media, and bone-related pathologies.

Zhang et al. (2015) showed that MRI was significantly more advantageous in diagnosing multiple cerebral infarcts in middle-aged patients in comparison with the findings of CT scan [19]. It was shown that MRI findings related to infraction at parietal and frontal areas, thalamus and basal ganglia were significantly more effective in the diagnosis of neurological disorders in comparison with the findings of CT scan. These results were consistent with the results obtained in the current study. Deficit-related symptoms, including aphasia, consciousness disorder and hemiplegia, help in identifying the site of cerebral infarction, thereby they provide the basis for initial diagnosis. It has been shown that findings of CT and MRI are useful in distinguishing pathological tissues from normal tissues, especially the brain tissues. However, the results of the current study have reported that MRI is the most suitable diagnostic imaging tool that helped neurologists determine the actual cause and condition of neurologi- 
cal diseases. A recent study conducted by Kenteu et al. (2018) has shown that the neuroimaging techniques including CT and MRI were effective in detecting tissue perfusion, strokes and vessel patency [20].

It was believed that the current study significantly contributes to the assessment of the effectiveness of both techniques in diagnosing neurological disorders. Improvement in patient outcomes depends on the imaging tool used in the effective diagnosis and treatment of neurological disorders. The findings of the current study not only enhance the understanding of pathological processes but also pave the way towards development of effective treatment strategies for neurological disorders. The current study has demonstrated that these techniques are effective in the diagnosis and treatment of several neurologic disorders and that it is impossible to provide specific treatment of strokes without any radiological diagnosis. Visual impairment, based on the present study findings, has also been reported to be highest as patients experienced from nystagmus, blurring of vision, cortical blindness and hemianopia.

The study did not conduct direct comparison between the findings of MRI and CT scan, which is a major study limitation. Moreover, the study did not discuss the preventive strategies adopted by clinicians to provide quality neurological care to patients. Studies are also required to assess preventive strategies and practices to be adopted by neurologists to provide quality neurological care to patients.

\section{Conclusion}

The current study has evaluated the findings of CT scan and MRI for diagnosing neurological disturbances. The study findings revealed that unlike CT scan, MRI was more successful in diagnosing different neurological conditions. Therefore, this study claims that MRI is an integrated diagnostic approach for assessing and dragonizing the neurological disorders; this study also serves as a valuable source for advancing the current neurological understanding of major diseases associated with the central nervous system. Therefore, future studies are required to perform direct comparison between MRI and CT scan to find out which technique is more effective. Moreover, other advanced imaging techniques should also be included in this comparison such as nuclear medicine imaging.

\section{Data Availability}

The data used to support the findings of this study are included within the article.

\section{Funding Statement}

There is no financial support and disclosure for the current study.

\section{Acknowledgements}

The author is thankful to all the associated personnel in any reference who con- 
tributed to the purpose of this research.

\section{Conflicts of Interest}

The author declares that there is no conflict of interest regarding the publication of this paper.

\section{References}

[1] Wingerchuk, D.M. and Carter, J.L. (2014) Multiple Sclerosis: Current and Emerging Disease-Modifying Therapies and Treatment Strategies. Mayo Clinic Proceedings, 89, 225-240. https://doi.org/10.1016/j.mayocp.2013.11.002

[2] Siuly, S. and Zhang, Y. (2016) Medical Big Data: Neurological Diseases Diagnosis through Medical Data Analysis. Data Science and Engineering, 1, 54-64. https://doi.org/10.1007/s41019-016-0011-3

[3] Roentgen, W.C. (2012) Wilhelm Conrad Röntgen: About a New Kind of Rays. Three Essays on the Discovery of X-Rays. Severus Publishing House, York.

[4] Leandrou, S. (2010) Magnetic Resonance Imaging and Computed Tomography in the Evaluation of Stroke. Dissertation Submitted in Partial Fulfilment for the Degree of MSc in Diagnostic Imaging.

[5] Rai, G.S., Rai, T., Jain, L., Vyas, M.M. and Roshan, R. (2016) Evaluation of CT and MRI Findings among Patients Presented with Chief Complaint of Headache in Central India. Journal of Clinical and Diagnostic Research, 10, TC21-TC25. https://doi.org/10.7860/JCDR/2016/16852.7249

[6] Fan, J.D., Yu, J.M. and Shao, Y. (2013) The Research Progress of Imaging Diagnostic of Hemorrhagic Infarction. Chinese Journal of Clinical Psychology, 19, 65-66.

[7] Jindal, M.A., Gaikwad, H.S., Hasija, B.D. and Vani, K. (2016) Comparison of Neuroimaging by CT and MRI and Correlation with Neurological Presentation in Eclampsia. International Journal of Reproduction, Contraception, Obstetrics and Gynecology, 2, 83-87.

[8] Gao, H. and Jiang, X. (2013) Progress on the Diagnosis and Evaluation of Brain Tumors. Cancer Imaging, 13, 466-481. https://dx.doi.org/10.1102\%2F1470-7330.2013.0039

[9] Meijer, F.J., Goraj, B., Bloem, B.R. and Esselink, R.A. (2017) Clinical Application of Brain MRI in the Diagnostic Work-Up of Parkinsonism. Journal of Parkinson Disease, 7, 211-217. https://doi.org/10.3233/jpd-150733

[10] Purandare, N.C. (2011) Inclusion of Brain in FDG PET/CT Scanning Techniques in Cancer Patients: Does It Obviate the Need for Dedicated Brain Imaging? Indian Journal of Nuclear Medicine, 26, 64-66. https://doi.org/10.4103/0972-3919.90253

[11] Goadsby, P.J. (2004) To Scan or Not to Scan in Headache. British Medical Journal, 329, 469-470. https://doi.org/10.1136/bmj.329.7464.469

[12] Clayton, J.A. (2016) Sex Influences in Neurological Disorders: Case Studies and Perspectives Dialogues. Journal of Clinical Neuroscience, 8, 357-360.

https://doi.org/10.31887/DCNS.2016.18.4/jclayton

[13] Liu, J., Qin, W., Nan, J., Li, J., Yuan, K., Zhao, L., et al. (2011) Gender-Related Differences in the Dysfunctional Resting Networks of a Migraine Suffers. PLoS ONE, 6, e27049. https://doi.org/10.1371/journal.pone.0027049

[14] Medvid, R., Ruiz, A., Komotar, R.J., Jagid, J.R., Ivan, M.E., Quencer, R.M., et al. (2015) Current Applications of MRI-Guided Laser Interstitial Thermal Therapy in 
the Treatment of Brain Neoplasms and Epilepsy: A Radiologic and Neurosurgical Overview. American Journal of Neuroradiology, 36, 1998-2006.

https://doi.org/10.3174/ajnr.A4362

[15] Holle, D. and Obermann, M. (2013) The Role of Neuroimaging in the Diagnosis of Headache Disorders. Therapeutic Advances in Neurological Disorders, 6, 369-374. https://doi.org/10.1177\%2F1756285613489765

[16] Nagahama, Y., Joshi, C., Dlouhy, B., Wu, A.Y., Abel, T.J., Baumbach, G., et al. (2017) Functional Hemispherotomy in Rasmussen Syndrome in the Absence of Classic MRI Findings. Epilepsy \& Behavior Case Reports, 7, 24-27.

https://doi.org/10.1016/j.ebcr.2016.11.003

[17] Degnan, A.J. and Levy, L.M. (2011) Pseudotumor Cerebri: Brief Review of Clinical Syndrome and Imaging Findings. American Journal of Neuroradiology, 32, 1986-1993. https://doi.org/10.3174/ajnr.A2404

[18] Aygun, D. and Bildik, F. (2003) Clinical Warning Criteria in Evaluation by Computed Tomography the Secondary Neurological Headaches in Adults. European Journal of Neurology, 10, 437-442. https://doi.org/10.1046/j.1468-1331.2003.00645.x

[19] Zhang, M.J., Zhang, X. and Xu, Y.X. (2015) Analysis on Value of CT and MRI Clinical Application in Diagnosis of Middle-Aged Patients with Multiple Cerebral Infarction. International Journal of Clinical and Experimental Medicine, 8, 17123-17127.

[20] Kenteu, B., Fogang, Y.F., Nyaga, U.F., Zafack, J.G., Jacques Noubiap, J. and Kamtchum-Tatuene, J. (2018) Neuroimaging of Headaches in Patients with Normal Neurological Examination: Protocol for a Systematic Review. BMJ Open, 8, Article ID: e020190. https://doi.org/10.1136/bmjopen-2017-020190 